\begin{tabular}{|c|l|}
\hline Title & On the special values of certain L-series related to half-integral weight modular forms \\
\hline Author(s) & Katsurada, Hidenori \\
\hline Citation & Hokkaido University Preprint Series in Mathematics, 1050, 1-25 \\
\hline Issue Date & 2014-3-17 \\
\hline DOI & 10.14943/84194 \\
\hline Doc URL & http://hdl.handle.net/2115/69854 \\
\hline Type & bulletin (article) \\
\hline File Information & pre1050.pdf \\
\hline
\end{tabular}

Instructions for use 


\title{
On the special values of certain L-series related to half-integral weight modular forms
}

\author{
Hidenori Katsurada*
}

\begin{abstract}
Let $h$ be a cuspidal Hecke eigenform of half-integral weight, and $E_{n / 2+1 / 2}$ be Cohen's Eisenstein series of weight $n / 2+1 / 2$. For a Dirichlet character $\chi$ we define a certain linear combination $R^{(\chi)}\left(s, h, E_{n /+1 / 2}\right)$ of the Rankin-Selberg convolution products of $h$ and $E_{n / 2+1 / 2}$ twisted by Dirichlet characters related with $\chi$. We then prove a certain algebraicity result for $R^{(\chi)}\left(l, h, E_{n / 2+1 / 2}\right)$ with $l$ integers.
\end{abstract}

\section{Introduction}

For two modular forms $h_{1}(z)$ and $h_{2}(z)$ of half-integral weights $k_{1}+1 / 2$ and $k_{2}+1 / 2$, respectively, for $\Gamma_{0}(4)$, and a primitive character $\chi$ we define the Rankin-Selberg convolution product $\widetilde{R}\left(s, h_{1}, h_{2}, \chi\right)$ twisted by $\chi$ as

$$
\widetilde{R}\left(s, h_{1}, h_{2}, \chi\right)=L\left(2 s-k_{1}-k_{2}+1, \chi_{-1}^{k_{1}-k_{2}} \chi^{2}\right) \sum_{m=1}^{\infty} \frac{c_{1}(m) c_{2}(m) \chi(m)}{m^{s}},
$$

where $c_{1}(m)$ and $c_{2}(m)$ denote the $m$-th Fourier coefficients of $h_{1}$ and $h_{2}$, respectively, and $L\left(s, \chi_{-1}^{k_{1}-k_{2}} \chi^{2}\right)$ is the Dirichlet $L$-function for $\chi_{-1}^{k_{1}-k_{2}} \chi^{2}$ (for the precise definition of $\chi_{-1}$ see Section 1.)

*This paper will appear in Proceedings in Mathematics and Statistics, Springer. The author was partly supported by Grant-in-Aid for Scientific Research, JSPS.

2000 Mathematics Subject Classification. Primary 11F67, 11F46, 11F66. 
The analytic properties of this Dirichlet series were investigated by Shimura [Sh2]. Furthermore the algebraicity of the values of this Dirichlet series at half-integers was deeply investigated by Shimura [Sh2]. However, as far as we know, there is no literature on the algebraicity of its special values at integers except for $[\mathrm{K}-\mathrm{M}]$. Therefore we naturally ask the following question:

Question. What can one say about the algebraicity of $\widetilde{R}\left(m, h_{1}, h_{2}, \chi\right)$ with $m$ an integer?

In $[\mathrm{K}-\mathrm{M}]$, we gave a partial answer to the above question in the case $h_{1}$ is a cuspidal Hecke eigenform in Kohnen's plus subspace for $\Gamma_{0}(4)$ and $h_{2}$ is Zagier's Eisenstein series of weight $3 / 2$. In this paper, we consider the above question in the case $h_{1}$ is a cuspidal Hecke eigenform in Kohnen's plus subspace for $\Gamma_{0}(4)$ and $h_{2}$ is Cohen's Eisenstein series. This paper is a summary of our paper $[\mathrm{Ka}]$, which will be published elsewhere. To state our main result more explicitly, we define another Dirichlet series $R\left(s, h_{1}, h_{2}, \chi\right)$ by

$$
R\left(s, h_{1}, h_{2}, \chi\right)=L\left(2 s-k_{1}-k_{2}+1, \chi^{2}\right) \sum_{m=1}^{\infty} c_{h_{1}}(m) c_{h_{2}}(m) \chi(m) m^{-s} .
$$

Assume that $k_{1}+k_{2}$ is even, and that the conductor of $\chi$ is odd. Then, as will be explained in Section 1, it suffices to consider the above question for $R\left(m, h_{1}, h_{2}, \chi\right)$ with integer $m$. Now let $k$ and $n$ be even integers such that $n \geq 4$ and $2 k-n \geq 12$. Let $h$ be a Hecke eigenform of weight $k-n / 2+1 / 2$ for $\Gamma_{0}(4)$ belonging to Kohnen's plus subspace, and $S(h)$ the normalized Hecke eigenform of weight $2 k-n$ for $S L_{2}(\mathbf{Z})$ corresponding to $h$ under the Shimura correspondence. Moreover let $E_{n / 2+1 / 2}$ be Cohen's Eisenstein series of weight $n / 2+1 / 2$ (for the precise definition of $E_{n / 2+1 / 2}$, see Section 2). Let $\chi$ be a primitive character of conductor $N$. We assume that $N$ is square free and let $N=p_{1} \cdots p_{r}$ be the prime decomposition of $N$. Put $l_{j}=l_{n, p_{j}}=$ G.C.D $\left(n, p_{j}-1\right)$. For an $r$-tuple $\left(i_{1}, i_{2}, \cdots, i_{r}\right)$ of integers put

$$
\chi_{\left(i_{1}, \cdots, i_{r}\right)}=\chi \prod_{j=1}^{r}\left(\frac{*}{p_{j}}\right)_{l_{j}}^{i_{j}}
$$

where $\left(\frac{*}{p_{j}}\right)_{l_{j}}$ denotes the $l_{j}$-th power residue symbol mod $p_{j}$. For two Dirich- 
let characters $\eta_{1}$ and $\eta_{2} \bmod N$, we define $J_{m}\left(\eta_{1}, \eta_{2}\right)$ by

$$
J_{m}\left(\eta_{1}, \eta_{2}\right)=\sum_{Z} \eta_{1}(\operatorname{det} Z) \eta_{2}(1-\operatorname{tr}(Z))
$$

where $Z$ runs over all symmetric matrices of degree $m$ with entries in $\mathbf{Z} / N \mathbf{Z}$ and $\operatorname{tr}(Z)$ denotes the trace of a matrix $Z$. We note that $J_{1}\left(\eta_{1}, \eta_{2}\right)$ is the Jacobi sum $J\left(\eta_{1}, \eta_{2}\right)$ associated with $\eta_{1}$ and $\eta_{2}$. We also put $J_{m}\left(\eta_{1}\right)=J_{m}\left(\eta_{1}\left(\frac{*}{N}\right)^{m-1}, \eta_{1}\right)$, where $\left(\frac{*}{N}\right)$ is the Jacobi symbol. We then define

$$
\begin{aligned}
& R^{(\chi)}\left(s, h, E_{n / 2+1 / 2}\right)=\sum_{i_{1}=0}^{l_{1}-1} \cdots \sum_{i_{r}=0}^{l_{r}-1} \overline{\chi_{\left(i_{1}, \cdots, i_{r}\right)}\left(2^{n}\right)} R\left(s, h, E_{n / 2+1 / 2}, \chi_{\left(i_{1}, \ldots, i_{r}\right)}\right) \\
& \times \overline{J\left(\chi_{\left(i_{1}, \cdots, i_{r}\right)},\left(\frac{*}{N}\right)\right)} \overline{J_{n-1}\left(\chi_{\left(i_{1}, \cdots, i_{r}\right)}\right)} \prod_{j=1}^{n / 2-1} L\left(2 s-2 j, S(h), \chi_{\left(i_{1}, \cdots, i_{r}\right)}^{2}\right),
\end{aligned}
$$

where $L\left(s, S(h), \chi_{\left(i_{1}, \cdots, i_{r}\right)}^{2}\right)$ is Hecke's $L$-function of $S(h)$ twisted by $\chi_{\left(i_{1}, \cdots, i_{r}\right)}^{2}$. Then our main result (Theorem 2.1) can be stated as follows: that

There exists a finite dimensional $\overline{\mathbf{Q}}$-vector space $W_{h, E_{n / 2+1 / 2}}$ in $\mathbf{C}$ such

$$
\frac{R^{(\chi)}\left(m, h, E_{n / 2+1 / 2}\right)}{\pi^{m n}} \in W_{h, E_{n / 2+1 / 2}}
$$

for any integer $m$ such that $n / 2+1 \leq m \leq k-n / 2-1$ and a character $\chi$ of odd square free conductor such that $\chi^{n}$ is primitive.

From the above result we easily obtain the following (cf. Theorem 2.2):

Let $r>\operatorname{dim}_{\overline{\mathbf{Q}}} W_{h, E_{n / 2+1 / 2}}$. Let $m_{1}, m_{2}, \cdots, m_{r}$ be integers such that $n / 2+$ $1 \leq m_{1}, m_{2}, \cdots, m_{r} \leq k-n / 2-1$ and $\chi_{1}, \chi_{2}, \cdots, \chi_{r}$ be Dirichlet characters of odd square free conductors $N_{1}, N_{2}, \cdots, N_{r}$, respectively such that $\chi_{i}^{n}$ is primitive for any $i=1,2, \cdots r$. Then the values

$\frac{R^{\left(\chi_{1}\right)}\left(m_{1}, h, E_{n / 2+1 / 2}\right)}{\pi^{m_{1} n}}, \cdots, \frac{R^{\left(\chi_{r}\right)}\left(m_{r}, h, E_{n / 2+1 / 2}\right)}{\pi^{m_{r} n}}$

are linearly dependent over $\overline{\mathbf{Q}}$.

This is a certain generalization of a main result in $[\mathrm{K}-\mathrm{M}]$ as will be explained later. 
A main tool for proving Theorem 2.1 is the twisted Koecher-Maaß series of the Duke-Imamoglu-Ikeda lift of $h$. To explain this, we define the twisted Koecher-Maaß series of a Siegel modular form in a more general setting. Let $F(Z)$ be a modular form of weight $k$ with respect to the symplectic group $S p_{n}(\mathbf{Z})$. For a positive integer $N$ let $S L_{n, N}(\mathbf{Z})=\left\{U \in S L_{n}(\mathbf{Z}) \mid U \equiv\right.$ $\left.1_{n} \bmod N\right\}$, and $e_{N}(T)=\#\left\{U \in S L_{n, N}(\mathbf{Z}) \mid T[U]=T\right\}$. For a primitive Dirichlet character $\chi$ mod $N$ we define the Koecher-Maaß series $L(s, F, \chi)$ of $F$ twisted by $\chi$ as

$$
L(s, F, \chi)=\sum_{T} \frac{\chi(\operatorname{tr}(T)) c_{F}(T)}{e_{N}(T)(\operatorname{det} T)^{s}},
$$

where $T$ runs over a complete set of representatives of $S L_{n, N}(\mathbf{Z})$-equivalence classes of positive definite half-integral matrices of degree $n$, and $c_{F}(T)$ denotes the $T$-th Fourier coefficient of $F$. We note that this Dirichlet series coincides with the Hecke $L$-function associated to $F$ twisted by $\chi$ in case $n=1$. Though we are mainly concerned with $L(s, F, \chi)$ in this paper, we also define another type of twisted Koecher-Maaß series $L^{*}(s, F, \chi)$ as

$$
L^{*}(s, F, \chi)=\sum_{T} \frac{\chi(\operatorname{det}(2 T)) c_{F}(T)}{e(T)(\operatorname{det} T)^{s}},
$$

where $T$ runs over a complete set of representatives of $S L_{n}(\mathbf{Z})$-equivalence classes of positive definite half-integral matrices of degree $n$, and $e(T)=$ $e_{1}(T)$. These two Dirichlet series $L(s, F, \chi)$ and $L^{*}(s, F, \chi)$ essentially coincide with each other in case $n=1$, but they don't in general. To distinguish these two Dirichlet series, we sometimes call $L(s, F, \chi)$ and $L^{*}(s, F, \chi)$ the twisted Koecher-Maaß series of the first and second kind, respectively. In Section 3, we will discuss a relation between these two Dirichlet series (cf. Theorem 3.5.) Now for the integers $k$ and $n$ stated above, let $h$ a cuspidal Hecke eigenform $h$ in Kohnen's plus subspace of weight $k-n / 2+1 / 2$ for $\Gamma_{0}(4)$. Let $I_{n}(h)$ be the Duke-Imamoglu-Ikeda lift of $h$ to the space of Siegel cusp forms of degree $n$. Then, in Section 4, first we give an explicit formula of $L^{*}\left(s, I_{n}(h), \eta\right)$ in terms of the Rankin-Selberg series $R\left(s, h, E_{n / 2+1 / 2}, \eta\right)$ and shifted products of Hecke's $L$-functions of $S(h)$ twisted by $\eta^{2}$ in the case $\eta$ is a primitive character (cf. Theorem 4.1.) Next, by this result combined with Theorem 3.5, we give an explicit formula of $L\left(s, I_{n}(h), \chi^{n}\right)$ in terms of $R^{(\chi)}\left(s, h, E_{n / 2+1 / 2}\right)$ and a sum of the shifted products $\prod_{j=1}^{n / 2-1} L\left(2 s-2 j+1, S(h), \chi_{\left(i_{1}, \cdots, i_{r}\right)}^{2}\right.$ ) (cf. 
Theorem 4.2 and its corollary.) This implies that $R^{(\chi)}\left(s, h, E_{n / 2+1 / 2}\right)$ can be expressed in terms of $L\left(s, I_{n}(h), \chi^{n}\right)$ and the sum of the shifted products. Thus we can prove our main result using the algebraicity of Hecke's $L$-function of $S(h)$ (cf. Theorem 1.1) combined with the arithmetic properties of $L\left(s, I_{n}(h), \chi^{n}\right)$, which were investigated by Choie and Kohnen [C-K] in a more general setting (cf. Theorem 3.2). We can also prove a functional equation for $R^{(\chi)}\left(s, h, E_{n / 2+1 / 2}\right)$ in case $n \equiv 2 \bmod 4$ using the functional equation for $L\left(s, F, \chi^{n}\right)$ (cf. Theorem 2.3.)

Notation. We denote by $\mathbf{e}(x)=\exp (2 \pi \sqrt{-1} x)$ for a complex number $x$. For a commutative ring $R$, we denote by $M_{m n}(R)$ the set of $(m, n)$ matrices with entries in $R$. For an $(m, n)$-matrix $X$ and an $(m, m)$-matrix $A$, we write $A[X]={ }^{t} X A X$, where ${ }^{t} X$ denotes the transpose of $X$. Let $a$ be an element of $R$. Then for an element $X$ of $M_{m n}(R)$ we often use the same symbol $X$ to denote the coset $X \bmod a M_{m n}(R)$. Put $G L_{m}(R)=\{A \in$ $\left.M_{m}(R) \mid \operatorname{det} A \in R^{*}\right\}$, and $S L_{m}(R)=\left\{A \in M_{m}(R) \mid \operatorname{det} A=1\right\}$, where $\operatorname{det} A$ denotes the determinant of a square matrix $A$ and $R^{*}$ is the unit group of $R$. We denote by $S_{n}(R)$ the set of symmetric matrices of degree $n$ with entries in $R$. In particular, if $S$ is a subset of $S_{n}(\mathbf{R})$ with $\mathbf{R}$ the field of real numbers, we denote by $S_{>0}$ (resp. $S_{\geq 0}$ ) the subset of $S$ consisting of positive definite (resp. semi-positive definite) matrices. The group $S L_{n}(\mathbf{Z})$ acts on the set $S_{n}(\mathbf{R})$ in the following way:

$$
S L_{n}(\mathbf{Z}) \times S_{n}(\mathbf{R}) \ni(g, A) \longrightarrow{ }^{t} g A g \in S_{n}(\mathbf{R}) .
$$

Let $G$ be a subgroup of $G L_{n}(\mathbf{Z})$. For a subset $\mathcal{B}$ of $S_{n}(\mathbf{R})$ stable under the action of $G$ we denote by $\mathcal{B} / G$ the set of equivalence classes of $\mathcal{B}$ with respect to $G$. We sometimes identify $\mathcal{B} / G$ with a complete set of representatives of $\mathcal{B} / G$. Two symmetric matrices $A$ and $A^{\prime}$ with entries in $R$ are said to be equivalent with each other with respect to $G$ and write $A \sim_{G} A^{\prime}$ if there is an element $X$ of $G$ such that $A^{\prime}=A[X]$. Let $\mathcal{L}_{n}$ denote the set of half-integral matrices of degree $n$ over $\mathbf{Z}$, that is, $\mathcal{L}_{n}$ is the set of symmetric matrices of degree $n$ whose $(i, j)$-component belongs to $\mathbf{Z}$ or $\frac{1}{2} \mathbf{Z}$ according as $i=j$ or not. 


\section{Review on the algebraicity of L-values of elliptic modular forms of integral and half- integral weights}

Before stating our main results, we review on the special values of $\mathrm{L}$ functions of elliptic modular forms of integral and half-integral weights. Put $J_{n}=$ $\left(\begin{array}{cc}O_{n} & -1_{n} \\ 1_{n} & O_{n}\end{array}\right)$, where $1_{n}$ and $O_{n}$ denotes the unit matrix and the zero matrix of degree $n$, respectively. Furthermore, put

$$
S p_{n}(\mathbf{Z})=\left\{M \in G L_{2 n}(\mathbf{Z}) \mid J_{n}[M]=J_{n}\right\} .
$$

Let $l$ be an integer or a half-integer, and let $\Gamma$ be a congruence subgroup of $S p_{n}(\mathbf{Z})$. We then denote by $M_{l}(\Gamma)$ the space of modular forms of weight $l$ with respect to $\Gamma$, and by $S_{l}(\Gamma)$ the subspace of $M_{l}(\Gamma)$ consisting of cusp forms. We also denote by $\Gamma_{0}(4)$ the subgroup of $S L_{2}(\mathbf{Z})$ consisting of matrices whose left lower entries are congruent to $0 \bmod N$. Let

$$
f(z)=\sum_{m=1}^{\infty} c_{f}(m) \mathbf{e}(m z)
$$

be a normalized Hecke eigenform in $S_{k}\left(S L_{2}(\mathbf{Z})\right)$, and $\chi$ be a primitive Dirichlet character. Then let us define Hecke's $L$-function $L(s, f, \chi)$ of $f$ twisted by $\chi$ as

$$
L(s, f, \chi)=\sum_{m=1}^{\infty} c_{f}(m) \chi(m) m^{-s} .
$$

Then we have the following result (cf. [Sh1]):

Theorem 1.1 There exist complex numbers $u_{ \pm}(f)$ uniquely determined up to $\overline{\mathbf{Q}}^{\times}$multiple such that

$$
L(m, f, \chi)\left(\pi^{m} u_{j}(f)\right)^{-1} \in \overline{\mathbf{Q}}
$$

for any integer $0<m \leq k-1$ and a primitive character $\chi$, where $j=+$ or - according as $(-1)^{m} \chi(-1)=1$ or -1 .

We remark that we have $L(m, f, \chi) \neq 0$ if $m \neq k / 2$, and $L(k / 2, f, \chi) \neq 0$ for infinitely many $\chi$. 
Next let us consider the half-integral weight case. Let

$$
h_{1}(z)=\sum_{m=1}^{\infty} c_{h_{1}}(m) \mathbf{e}(m z)
$$

be a Hecke eigenform in $S_{k_{1}+1 / 2}\left(\Gamma_{0}(4)\right)$, and

$$
h_{2}(z)=\sum_{m=0}^{\infty} c_{h_{2}}(m) \mathbf{e}(m z)
$$

be an element of $M_{k_{2}+1 / 2}\left(\Gamma_{0}(4)\right)$. For positive integers $e$ and $l$, let $\chi_{(-1)^{l} e}$ be the Dirichlet character corresponding to the extension $\mathbf{Q}\left(\sqrt{(-1)^{l} e} / \mathbf{Q}\right)$. Let $\chi$ be a primitive character $\bmod N$. Then we define

$$
\widetilde{R}\left(s, h_{1}, h_{2}, \chi\right)=L\left(2 s-k_{1}-k_{2}+1, \omega\right) \sum_{m=1}^{\infty} c_{h_{1}}(m) c_{h_{2}}(m) \chi(m) m^{-s},
$$

where $\omega(d)=\chi_{-1}^{k_{1}-k_{2}} \chi^{2}(d)$. Now let $S\left(h_{1}\right)$ be the normalized Hecke eigenform in $S_{2 k_{1}}\left(S L_{2}(\mathbf{Z})\right)$ corresponding to $h_{1}$ under the Shimura correspondence. Then the following result is due to Shimura [Sh2].

Theorem 1.2 Assume that $k_{1}>k_{2}$. Under the above notation we have

$$
\widetilde{R}\left(m+1 / 2, h_{1}, h_{2}, \chi\right)\left(u_{-}\left(S\left(h_{1}\right)\right) \pi^{-k_{2}+1+2 m}\right)^{-1} \in \overline{\mathbf{Q}}\left(h_{1}\right) \overline{\mathbf{Q}}\left(h_{2}\right)
$$

for any integer $k_{2} \leq m \leq k_{1}-1$ and a primitive character $\chi$, where $\overline{\mathbf{Q}}\left(h_{i}\right)$ is the field, generated over $\overline{\mathbf{Q}}$, by all the Fourier coefficients of $h_{i}$.

Corollary Let the notation be as above. Assume that $k_{1}>k_{2}$ and that $c_{h_{1}}(n), c_{h_{2}}(n) \in \overline{\mathbf{Q}}$ for any $n \in \mathbf{Z}_{\geq 0}$. Then there exists a one-dimensional $\overline{\mathbf{Q}}$-vector space $U_{h_{1}, h_{2}}$ in $\mathbf{C}$ such that

$$
\widetilde{R}\left(m+1 / 2, h_{1}, h_{2}, \chi\right) \pi^{-2 m} \in U_{h_{1}, h_{2}}
$$

for any integer $k_{2} \leq m \leq k_{1}-1$ and a primitive character $\chi$.

Now we consider the values of $\widetilde{R}\left(s, h_{1}, h_{2}, \chi\right)$ at integers. Let

$$
R\left(s, h_{1}, h_{2}, \chi\right)=L\left(2 s-k_{1}-k_{2}+1, \chi^{2}\right) \sum_{m=1}^{\infty} c_{h_{1}}(m) c_{h_{2}}(m) \chi(m) m^{-s} .
$$


be the Dirichlet series defined in Section 0. Assume that $k_{1}+k_{2}$ is even, and that the conductor of $\chi$ is odd. Then we have

$$
R\left(s, h_{1}, h_{2}, \chi\right)=\left(1-2^{-2 s+k_{1}+k_{2}-1} \chi^{2}(2)\right)^{-1} \widetilde{R}\left(s, h_{1}, h_{2}, \chi\right) .
$$

Hence it suffices to consider the question in Section 0 for $R\left(m, h_{1}, h_{2}, \chi\right)$ with integer $m$.

\section{Main results}

For a non-negative integer $m$ and a positive integer $l$, Cohen's function $H(l, m)$ is given by $H(l, m)=L_{-m}(1-l)$. Here

$$
\begin{aligned}
L_{D}(s) & \left\{\begin{aligned}
\zeta(2 s-1), & D=0 \\
L\left(s, \chi_{D_{K}}\right) \sum_{a \mid f} \mu(a) \chi_{D_{K}}(a) a^{-s} \sigma_{1-2 s}(f / a), & D \neq 0, D \equiv 0,1 \bmod 4 \\
0, & D \equiv 2,3 \bmod 4,
\end{aligned}\right.
\end{aligned}
$$

where the positive integer $f$ is defined by $D=D_{K} f^{2}$ with the discriminant $D_{K}$ of $K=\mathbf{Q}(\sqrt{D}), \chi_{D_{K}}$ is the Kronecker symbol, $\mu$ is the Möbius function and $\sigma_{s}(n)=\sum_{d \mid n} d^{s}$. Furthermore we define Cohen's Eisenstein series $E_{l+1 / 2}(z)$ by

$$
E_{l+1 / 2}(z)=\sum_{m=0}^{\infty} H(l, m) \mathbf{e}(m z) .
$$

It is known that $E_{l+1 / 2}(z)$ is a modular form of weight $l+1 / 2$ belonging to Kohnen's plus space. Let $k$ and $n$ be positive even integers such that $n \geq 4,2 k-n \geq 12$. Let $h(z)$ be a Hecke eigenform in Kohnen's plus subspace $S_{k-n / 2+1 / 2}^{+}\left(\Gamma_{0}(4)\right)$ (cf. [Ko]), and $S(h)$ be the normalized Hecke eigenform in $S_{2 k-n}\left(S L_{2}(\mathbf{Z})\right)$ corresponding to $h$ under the Shimura correspondence. Let $p$ be a prime number and $l$ be a positive integer dividing $p-1$. Take an $l$-th root of unity $\zeta_{l}$ and a prime ideal $\mathfrak{p}$ of $\mathbf{Q}\left(\zeta_{l}\right)$ lying above $p$. Let $a$ be an integer prime to $p$. Then we have $a^{(p-1) / l} \equiv \zeta_{l}^{i} \bmod \mathfrak{p}$ with some $i \in \mathbf{Z}$. We then put $\left(\frac{a}{p}\right)_{l}=\zeta^{i}$. We call $\left(\frac{*}{p}\right)_{l}$ the $l$-th power residue symbol mod $p$. In the case $l=2$, this is the Legendre symbol, and we write it as $\left(\frac{*}{p}\right)$ as usual. We 
note that this definition of the power residue symbol is different from the usual one, and depends on the choice of $\mathfrak{p}$ and $\zeta_{l}$ except the case $l=2$. We denote by $\left(\frac{*}{N}\right)$ the Jacobi symbol for a positive odd integer $M$. Let $\chi$ be a primitive Dirichlet character of conductor $N$. We assume that $N$ is a square free odd integer, and write $N=p_{1} \cdots p_{r}$ with $p_{1}, \cdots, p_{r}$ prime numbers. Put $l_{j}=l_{n, p_{j}}=$ G.C.D $\left(n, p_{j}-1\right)$. For an $r$-tuple $\left(i_{1}, i_{2}, \cdots, i_{r}\right)$ of integers put

$$
\chi_{\left(i_{1}, \cdots, i_{r}\right)}=\chi \prod_{j=1}^{r}\left(\frac{*}{p_{j}}\right)_{l_{j}}^{i_{j}} .
$$

For two Dirichlet characters $\eta_{1}$ and $\eta_{2} \bmod N$, let $J_{m}\left(\eta_{1}, \eta_{2}\right)$ and $J_{m}\left(\eta_{1}\right)$ be as those defined in Section 0. By definition, $J_{m}\left(\eta_{1}, \eta_{2}\right)$ is an algebraic number. As in Section 0, we define

$$
\begin{aligned}
& R^{(\chi)}\left(s, h, E_{n / 2+1 / 2}\right) \\
& =\sum_{i_{1}=0}^{l_{1}-1} \cdots \sum_{i_{r}=0}^{l_{r}-1} \overline{\chi_{\left(i_{1}, \cdots, i_{r}\right)}\left(2^{n}\right)} \overline{J\left(\chi_{\left(i_{1}, \cdots, i_{r}\right)},\left(\frac{*}{N}\right)\right)} \overline{J_{n-1}\left(\chi_{\left(i_{1}, \cdots, i_{r}\right)}\right)} \\
& \quad \times R\left(s, h, E_{n / 2+1 / 2}, \chi_{\left(i_{1}, \ldots, i_{r}\right)}\right) \mathbf{L}_{n}\left(s, S(h), \chi_{\left(i_{1}, \cdots, i_{r}\right)}\right),
\end{aligned}
$$

where

$$
\mathbf{L}_{n}(s, S(h), \eta)=\prod_{j=1}^{n / 2-1} L\left(2 s-2 j, S(h), \eta^{2}\right)
$$

for a primitive character $\eta$. We note that $R^{(\chi)}\left(s, h, E_{n / 2+1 / 2}\right)$ does not depend on the choice of an $l_{i}$-th root of unity $\zeta_{l_{i}}$ and an prime ideal $\mathfrak{p}_{i}$ of $\mathbf{Q}\left(\zeta_{l_{i}}\right)$ lying above $p_{i}$.

Remark. (1) Let $m$ be an integer s.t. $n / 2+1 \leq m \leq k-n / 2-1$. Then the value $\frac{\mathbf{L}_{n}\left(m, S(h), \chi_{\left(i_{1}, \cdots, i_{r}\right)}^{2}\right)}{\pi^{m(n-2)}}$ belongs to $\overline{\mathbf{Q}} u_{+}(S(h))^{n / 2-1} \pi^{-n^{2} / 4+n / 2}$ for any $\chi$. In particular if $n \equiv 2 \bmod 4$, then it is nonzero for any $\chi$, and if $n \equiv 0 \bmod 4$, then it is nonzero for infinitely many $\chi$.

(2) As will be stated in Section 3, $J_{n-1}\left(\chi_{\left(i_{1}, \cdots, i_{r}\right)}\right)$ is expressed as a product of Jacobi sums, and it is non-zero algebraic number if $\chi^{n}$ is rewrote.

Theorem 2.1 There exists a finite dimensional $\overline{\mathbf{Q}}$-vector space $W_{h, E_{n / 2+1 / 2}}$ in $\mathbf{C}$ such that

$$
\frac{R^{(\chi)}\left(m, h, E_{n / 2+1 / 2}\right)}{\pi^{m n}} \in W_{h, E_{n / 2+1 / 2}}
$$


for any integer $n / 2+1 \leq m \leq k-n / 2-1$ and a character $\chi$ of odd square free conductor such that $\chi^{n}$ is rewrote.

Theorem 2.2 Let $r>\operatorname{dim}_{\overline{\mathbf{Q}}} W_{h, E_{n / 2+1 / 2}}$. Let $m_{1}, m_{2}, \cdots, m_{r}$ be integers such that $n / 2+1 \leq m_{1}, m_{2}, \cdots, m_{r} \leq k-n / 2-1$ and $\chi_{1}, \chi_{2}, \cdots, \chi_{r}$ be Dirichlet characters of odd square free conductors $N_{1}, N_{2}, \cdots, N_{r}$, respectively such that $\chi_{i}^{n}$ is primitive for any $i=1,2, \cdots r$. Then the values $\frac{R^{\left(\chi_{1}\right)}\left(m_{1}, h, E_{n / 2+1 / 2}\right)}{\pi^{m_{1} n}}, \cdots, \frac{R^{\left(\chi_{r}\right)}\left(m_{r}, h, E_{n / 2+1 / 2}\right)}{\pi^{m_{r} n}}$ are linearly dependent over $\overline{\mathrm{Q}}$.

Corollary Assume that $n \equiv 2 \bmod 4$. Let $r$ and $m_{1}, m_{2}, \cdots, m_{r}$ be as above. Let $\chi_{1}, \chi_{2}, \cdots, \chi_{r}$ be Dirichlet characters of odd prime conductors $p_{1}, p_{2}, \cdots, p_{r}$, respectively such that $\chi_{i}^{n}$ is non-trivial for any $i=1,2, \cdots r$. Put $l_{i}=\operatorname{GCD}\left(n, p_{i}-1\right)$. Then the values $\left\{\frac{R\left(m_{i}, h, E_{n / 2+1 / 2}, \chi_{i(j)}\right)}{\pi^{2 m_{i}}}\right\}_{1 \leq i s}$

are linearly dependent over $\overline{\mathbf{Q}}$.

We also have a functional equation for $R^{(\chi)}\left(s, h, E_{n / 2+1 / 2}\right)$ :

Theorem 2.3 Let $h$ be as above. Let $\chi$ be a primitive character of odd square free conductor $N$. Assume that $n \equiv 2 \bmod 4$, and that $\chi^{n}$ is primitive. Put

$$
\mathcal{R}^{(\chi)}\left(s, h, E_{n / 2+1 / 2}\right)=N^{2 s} \tau\left(\chi^{n}\right)^{-1} \gamma_{n}(s) R^{(\chi)}\left(s, h, E_{n / 2+1 / 2}\right),
$$

where $\tau\left(\chi^{n}\right)$ is the Gauss sum of $\chi^{n}$, and

$$
\gamma_{n}(s)=(2 \pi)^{-n s} \prod_{i=1}^{n} \pi^{(i-1) / 2} \Gamma(s-(i-1) / 2) .
$$

Then $\mathcal{R}^{(\chi)}\left(s, h, E_{n / 2+1 / 2}\right)$ has an analytic continuation to the whole s-plane, and has the following functional equation:

$$
\mathcal{R}^{(\chi)}\left(k-s, h, E_{n / 2+1 / 2}\right)=\mathcal{R}^{(\chi)}\left(s, h, E_{n / 2+1 / 2}\right) .
$$

Remark. (1) The series $\left\{R\left(s, h, E_{n / 2+1 / 2}, \chi_{i(j)}\right)\right\}_{1 \leq i \leq r, 0 \leq j \leq l_{i}-1}$ are linearly independent over $\mathbf{C}$ as functions of $s$.

(2) In the case of $n=2$, this type of result was given for $R\left(m, h, E_{3 / 2}\right)$ with $E_{3 / 2}$ Zagier's Eisenstein series of weight $3 / 2$ by $[\mathrm{K}-\mathrm{M}]$. Cohen's Eisenstein 
series is a holomorphic modular form, where as Zagier's Eisenstein series is not. Nevertheless, the former can be regarded as a generalization of the latter. Therefore, our present result can be regarded as a generalization of $[\mathrm{K}-\mathrm{M}]$. (3) The meromorphy of this type of series was derived in [Sh2] by using so called the Rankin-Selberg integral expression in a more general setting, but we don't know whether the functional equation of the above type can be directly proved without using the above method.

\section{Twisted Koecher-Maaß series}

To prove the main results, in this section and the next, we consider the twisted Koecher-Maaß series of a Siegel modular form. Let $F(Z) \in M_{k}\left(S p_{n}(\mathbf{Z})\right)$. Then $F(Z)$ has the Fourier expansion:

$$
F(Z)=\sum_{T \in \mathcal{L}_{n} \geq 0} c_{F}(T) \mathbf{e}(\operatorname{tr}(T Z))
$$

where $\operatorname{tr}(X)$ denotes the trace of a matrix $X$. For $N \in \mathbf{Z}_{>0}$, put $S L_{n, N}(\mathbf{Z})=$ $\left\{U \in S L_{n}(\mathbf{Z}) \mid U \equiv 1_{n} \bmod N\right\}$, and for $T \in \mathcal{L}_{n>0}$ put $e_{N}(T)=\#\{U \in$ $\left.S L_{n, N}(\mathbf{Z}) \mid T[U]=T\right\}$. For a primitive Dirichlet character $\chi \bmod N$ Let

$$
L(s, F, \chi)=\sum_{T \in \mathcal{L}_{n>0} / S L_{n, N}(\mathbf{Z})} \frac{\chi(\operatorname{tr}(T)) c_{F}(T)}{e_{N}(T)(\operatorname{det} T)^{s}}
$$

be the twisted Koecher-Maaß series of $F$ of the first kind as in Section 0 . The following two theorems are due to Choie and Kohnen [C-K].

Theorem 3.1 Let $F \in S_{k}\left(S p_{n}(\mathbf{Z})\right)$, and $\chi$ a primitive character of conductor N. Put

$$
\gamma_{n}(s)=(2 \pi)^{-n s} \prod_{i=1}^{n} \pi^{(i-1) / 2} \Gamma(s-(i-1) / 2),
$$

and

$$
\Lambda(s, F, \chi)=N^{2 s} \tau(\chi)^{-1} \gamma_{n}(s) L(s, F, \chi) \quad(\operatorname{Re}(s)>>0),
$$

where $\tau(\chi)$ is the Gauss sum of $\chi$. Then $\Lambda(s, F, \chi)$ has an analytic continuation to the whole s-plane and has the following functional equation:

$$
\Lambda(k-s, F, \chi)=(-1)^{n k / 2} \chi(-1) \Lambda(s, F, \bar{\chi}) .
$$


Theorem 3.2 Let $F$ and $\chi$ be as above. Then there exists a finite dimensional $\overline{\mathbf{Q}}$-vector space $V_{F}$ in $\mathbf{C}$ such that

$$
L(m, F, \chi) \pi^{-n m} \in V_{F}
$$

for any primitive character $\chi$ and any integer $m$ such that $(n+1) / 2 \leq m \leq$ $k-(n+1) / 2$.

Example. Let $n=1$. Take a basis $\left\{f_{1}, \cdots, f_{d}\right\}$ of $S_{k}\left(S L_{2}(\mathbf{Z})\right)$ consisting of normalized Hecke eigenforms. Write $f \in S_{k}\left(S L_{2}(\mathbf{Z})\right)$ as

$$
f=a_{1} f_{1}+\cdots+a_{d} f_{d}
$$

with $a_{1}, \cdots, a_{d} \in \mathbf{C}$. Then put $w_{i}=a_{i} u_{+}\left(f_{i}\right), w_{d+i}=a_{i} u_{-}\left(f_{i}\right)(i=1, \cdots, d)$ and $V_{f}=\sum_{i=1}^{2 d} \overline{\mathbf{Q}} w_{i}$. Then $V_{f}$ satisfies the required property for $f$.

Now let

$$
L^{*}(s, F, \chi)=\sum_{T \in \mathcal{L}_{n>0} / S L_{n}(\mathbf{Z})} \frac{\chi(\operatorname{det}(2 T)) c_{F}(T)}{e(T)(\operatorname{det} T)^{s}}
$$

be the twisted Koecher-Maaß series of $F$ of the second kind as in Section 0 . We will discuss a relation between these two Dirichlet series. Let $N$ be a positive integer. Let $g$ be a periodic function on $\mathbf{Z}$ with a period $N$ and $\phi$ a polynomial in $t_{1}, \ldots, t_{r}$. Then for an element $u=\left(a_{1} \bmod N, \ldots, a_{r} \bmod N\right) \in$ $(\mathbf{Z} / N \mathbf{Z})^{r}$, the value $g\left(\phi\left(a_{1}, \ldots, a_{r}\right)\right)$ does not depend on the choice of the representative $u$. Therefore we denote this value by $g(\phi(u))$. Now let $\chi$ be a primitive character $\bmod N$. For $A \in \mathcal{L}_{n>0}$, put

$$
h(A, \chi)=\sum_{U \in S L_{n}(\mathbf{Z} / N \mathbf{Z})} \chi(\operatorname{tr}(A[U])) .
$$

The following proposition is due to [[K-M], Proposition 3.1].

\section{Proposition 3.3 Let}

$$
F(Z)=\sum_{A \in \mathcal{L}_{n \geq 0}} c_{F}(A) \mathbf{e}(\operatorname{tr}(A Z))
$$

be an element of $M_{k}\left(S p_{n}(\mathbf{Z})\right)$. Let $\chi$ be a Dirichlet character mod $N$. Assume $N \neq 2$. Then we have

$$
L(s, F, \chi)=\sum_{A \in \mathcal{L}_{n>0} / S L_{n}(\mathbf{Z})} \frac{c_{F}(A) h(A, \chi)}{e(A)(\operatorname{det} A)^{s}} .
$$


For a Dirichlet character $\chi \bmod N$, let $\chi^{(p)}$ be the $p$-factor of $\chi$ so that $\chi=\prod_{p \mid N} \chi^{(p)}$. For a prime number $p$ put

$$
\gamma_{n, p}=p^{n^{2}-n(n+1) / 2}\left(1-p^{-n / 2}\right) \prod_{e=1}^{(n-2) / 2}\left(1-p^{-2 e}\right)
$$

or

$$
\gamma_{n, p}=p^{n^{2}-n(n+1) / 2} \prod_{e=1}^{(n-1) / 2}\left(1-p^{-2 e}\right)
$$

according as $n$ is even or odd. The following result is a technical tool for proving our main result.

Theorem 3.4 Let $A \in \mathcal{L}_{n>0}$. Let $N$ be a square free odd integer, and let $N=\prod_{i=1}^{r} p_{i}$ be the prime decomposition of $N$. Let $\chi$ be a primitive Dirichlet character $\bmod N$. For each positive integer $i \leq r$, put $l_{i}=$ G.C.D $\left(n, p_{i}-1\right)$ and let $u_{0, i}$ be a primitive $l_{i}$-th root of unity $\bmod p_{i}$.

(1). If $\chi^{\left(p_{i}\right)}\left(u_{0, i}\right) \neq 1$ for some $i$. Then we have $h(A, \chi)=0$.

(2). Assume that $\chi^{\left(p_{i}\right)}\left(u_{0, i}\right)=1$ for any $i$. Fix a character $\tilde{\chi}$ such that $\tilde{\chi}^{n}=\chi$.

(2.1) Let $n$ be even. Then we have

$$
h(A, \chi)=\prod_{i=1}^{r}(-1)^{n\left(p_{i}-1\right) / 4} \gamma_{n, p_{i}}
$$

$\times \sum_{i_{1}=0}^{l_{1}-1} \cdots \sum_{i_{r}=0}^{l_{r}-1} \overline{\widetilde{\chi}}_{\left(i_{1}, \cdots, i_{r}\right)}\left(2^{n}\right) \widetilde{\chi}_{\left(i_{1}, \cdots, i_{r}\right)}(\operatorname{det}(2 A)) \overline{J\left(\widetilde{\chi}_{\left(i_{1}, \cdots, i_{r}\right)},\left(\frac{*}{N}\right)\right)} \overline{J_{n-1}\left(\widetilde{\chi}_{\left(i_{1}, \cdots, i_{r}\right)}\right)}$.

(2.2) Let $n$ be odd, and assume that $\chi^{2}$ is primitive. Then we have

$$
\begin{gathered}
h(A, \chi)=\prod_{i=1}^{r}(-1)^{(n-1)\left(p_{i}-1\right) / 4} \gamma_{n, p_{i}} \\
\times \sum_{i_{1}=0}^{l_{1}-1} \cdots \sum_{i_{r}=0}^{l_{r}-1} \overline{\widetilde{\chi}}_{\left(i_{1}, \cdots, i_{r}\right)}\left(2^{n}\right) \widetilde{\chi}_{\left(i_{1}, \cdots, i_{r}\right)}(\operatorname{det}(2 A)) \overline{J_{n-1}\left(\widetilde{\chi}_{\left(i_{1}, \cdots, i_{r}\right)}\right)} .
\end{gathered}
$$

The proof of the above theorem is elementary but is rather lengthy. The details will be given in $[\mathrm{Ka}]$. 
Remark. Let $\eta$ be a primitive Dirichlet character of odd prime conductor $p$. Assume that $\eta^{2} \neq 1$. Then we can prove that we have

$$
J\left(\eta,\left(\frac{*}{p}\right)\right) J\left(\eta\left(\frac{*}{p}\right), \eta\left(\frac{*}{p}\right)\right)=\left(\frac{-1}{p}\right) \bar{\eta}(4) p .
$$

(This is not so trivial. For the details, see [Ka].) Hence for $A \in \mathcal{L}_{2>0}$ and a primitive character $\chi$ of odd square free conductor $N$ such that $\chi^{(p)}(-1)=1$ for any prime divisor $p$ of $N$, we have

$$
\left.h(A, \chi)=\prod_{p \mid N}\left\{\left(1+\left(\frac{4 \operatorname{det} A}{p}\right)\right)\left(1-\left(\frac{-1}{p}\right) p^{-1}\right)\right\} N^{2}\left(\frac{-1}{N}\right) \tilde{\chi}(4 \operatorname{det} A)\right),
$$

where $\tilde{\chi}$ is a character such that $\tilde{\chi}^{2}=\chi$. This coincides with (2) of Theorem 3.8 in $[\mathrm{K}-\mathrm{M}]$.

By Theorem 3.4 and Proposition 3.3 we easily obtain:

Theorem 3.5 Let $N, p_{i}, l_{i}, u_{0, i}(i=1, \cdots, r)$ and $\chi$ be as in Theorem 3.4, and let $F$ be an element of $M_{k}\left(S p_{n}(\mathbf{Z})\right)$.

(1). If $\chi^{\left(p_{i}\right)}\left(u_{0, i}\right) \neq 1$ for some $i$. Then we have $L(s, F, \chi)=0$.

(2). Assume that $\chi^{\left(p_{i}\right)}\left(u_{0, i}\right)=1$ for any $i$. Fix a character $\tilde{\chi}$ such that $\tilde{\chi}^{n}=\chi$.

(2.1) Let $n$ be even. Then we have

$$
L(s, F, \chi)=\prod_{i=1}^{r}(-1)^{n\left(p_{i}-1\right) / 4} \gamma_{n, p_{i}}
$$

$\times \sum_{i_{1}=0}^{l_{1}-1} \cdots \sum_{i_{r}=0}^{l_{r}-1} \widetilde{\widetilde{\chi}}_{\left(i_{1}, \cdots, i_{r}\right)}\left(2^{n}\right) \overline{J\left(\widetilde{\chi}_{\left(i_{1}, \cdots, i_{r}\right)},\left(\frac{*}{N}\right)\right)} \overline{J_{n-1}\left(\widetilde{\chi}_{\left(i_{1}, \cdots, i_{r}\right)}\right)} L^{*}\left(s, F, \widetilde{\chi}_{\left(i_{1}, i_{2}, \cdots, i_{r}\right)}\right)$.

(2.2) Let $n$ be odd, and assume that $\chi^{2}$ is primitive. Then we have

$$
\begin{gathered}
L(s, F, \chi)=\prod_{i=1}^{r}(-1)^{(n-1)\left(p_{i}-1\right) / 4} \gamma_{n, p_{i}} \\
\times \sum_{i_{1}=0}^{l_{1}-1} \cdots \sum_{i_{r}=0}^{l_{r}-1} \overline{\widetilde{\chi}}_{\left(i_{1}, \cdots, i_{r}\right)}\left(2^{n}\right) \overline{J_{n-1}\left(\widetilde{\chi}_{\left(i_{1}, i_{2}, \cdots, i_{r}\right)}\right)} L^{*}\left(s, F, \widetilde{\chi}_{\left(i_{1}, i_{2}, \cdots, i_{r}\right)}\right) .
\end{gathered}
$$


To give an explicit formula of $J_{m}(\chi, \eta)$ for primitive characters $\chi, \eta \bmod$ $N$, we define $I_{m}(\chi, \eta)$ as

$$
I_{m}(\chi, \eta)=\sum_{Z \in S_{m}(\mathbf{Z} / N \mathbf{Z})} \chi(\operatorname{det} Z) \eta(\operatorname{tr}(Z)) .
$$

Then we have the following two propositions, whose proof will be given precisely in $[\mathrm{Ka}]$.

Proposition 3.6 Let $\chi$ and $\eta$ be primitive character mod an odd prime number $p$. Assume that $\chi^{2} \neq 1$ and that $\eta$ is non-trivial. Put $c_{m}(\chi, \eta)=1$ or 0 according as $\chi^{m-1} \eta=1$ or not.

(1) Assume that $m$ is odd. Then

$$
I_{m}(\chi, \eta)=c_{m}(\chi, \eta)\left(\frac{-1}{p}\right)^{(m-1) / 2} p^{(m-1) / 2}(p-1) J_{m-1}\left(\chi\left(\frac{*}{p}\right), \eta\right) .
$$

(2) Assume that $m$ is even. Then

$I_{m}(\chi, \eta)=c_{m}(\chi, \eta)\left(\frac{-1}{p}\right)^{m / 2} p^{(m-2) / 2}(p-1) \chi(-1) J\left(\chi,\left(\frac{*}{p}\right)\right) J_{m-1}\left(\chi\left(\frac{*}{p}\right), \eta\right)$.

Proposition 3.7 Let $\chi, \eta$ and $p$ be as in Proposition 3.6.

(1) Assume that $m$ is odd. Then

$$
\begin{gathered}
J_{m}(\chi, \eta)=\left(\frac{-1}{p}\right)^{(m-1) / 2} p^{(m-1) / 2} \\
\times\left\{J\left(\chi, \chi^{m-1} \eta\right) J_{m-1}\left(\chi\left(\frac{*}{p}\right), \eta\right)+\eta(-1) I_{m-1}\left(\chi\left(\frac{*}{p}\right), \eta\right)\right\} .
\end{gathered}
$$

(2) Assume that $m$ is even. Then

$$
\begin{gathered}
J_{m}(\chi, \eta)=\left(\frac{-1}{p}\right)^{m / 2} p^{(m-2) / 2} J\left(\chi,\left(\frac{*}{p}\right)\right) \\
\times\left\{J\left(\chi, \chi^{m-1}\left(\frac{*}{p}\right) \eta\right) J_{m-1}\left(\chi\left(\frac{*}{p}\right), \eta\right)+\eta(-1) I_{m-1}\left(\chi\left(\frac{*}{p}\right), \eta\right)\right\} .
\end{gathered}
$$

From the above two propositions we have the following: 
Theorem 3.8 Let $\chi$ be a primitive character with a prime conductor $p$ such that $\chi^{2} \neq 1$.

(1) Let $m$ be odd.

(1.1) Assume that $\chi^{m} \neq 1$. Then

$$
J_{m}\left(\chi\left(\frac{*}{p}\right)^{i}, \chi\right)=\left(\frac{-1}{p}\right)^{(m-1) / 2} p^{(m-1) / 2} J\left(\chi\left(\frac{*}{p}\right)^{i}, \chi^{m}\right) J_{m-1}\left(\chi\left(\frac{*}{p}\right)^{i+1}, \chi\right) .
$$

(1.2) Assume that $\chi^{m}=1$. Then

$$
J_{m}\left(\chi\left(\frac{*}{p}\right)^{i}, \chi\right)=p^{m-1}\left(\frac{-1}{p}\right)^{i+1} J\left(\chi\left(\frac{*}{p}\right)^{i+1},\left(\frac{*}{p}\right)\right) J_{m-2}\left(\chi\left(\frac{*}{p}\right)^{i}, \chi\right) .
$$

(2) Let $m$ be even.

(2.1) Assume that $\chi^{m}\left(\frac{*}{p}\right)^{i+1} \neq 1$. Then

$$
\begin{gathered}
J_{m}\left(\chi\left(\frac{*}{p}\right)^{i}, \chi\right) \\
=\left(\frac{-1}{p}\right)^{m / 2-1} J\left(\chi\left(\frac{*}{p}\right)^{i},\left(\frac{*}{p}\right)\right) J\left(\chi\left(\frac{*}{p}\right)^{i+1}, \chi^{m}\left(\frac{*}{p}\right)^{i+1}\right) J_{m-1}\left(\chi\left(\frac{*}{p}\right)^{i+1}, \chi\right) .
\end{gathered}
$$

(2.2) Assume that $\chi^{m}\left(\frac{*}{p}\right)^{i+1}=1$. Then

$$
J_{m}\left(\chi\left(\frac{*}{p}\right)^{i}, \chi\right)=\chi(-1) p^{m-1} J\left(\chi\left(\frac{*}{p}\right)^{i},\left(\frac{*}{p}\right)\right) J_{m-2}\left(\chi\left(\frac{*}{p}\right)^{i}, \chi\right) .
$$

Corollary Let $\chi$ be a primitive character with an odd square free conductor $N$. Assume that $\chi^{2}$ is primitive. Then the value $J_{m}(\chi)$ is nonzero.

\section{An explicit formula for the twisted Koecher- Maaß series of the D-I-I lift}

Throughout this section and the next, we assume that $n$ and $k$ are even positive integers. Let $h$ be a Hecke eigenform of weight $k-n / 2+1 / 2$ belonging to Kohnen's plus space. Then $h$ has the following Fourier expansion:

$$
h(z)=\sum_{e} c_{h}(e) \mathbf{e}(e z),
$$


where $e$ runs over all positive integers such that $(-1)^{k-n / 2} e \equiv 0,1 \bmod 4$. Let

$$
S(h)(z)=\sum_{m=1}^{\infty} c_{S(h)}(m) \mathbf{e}(m z)
$$

be the normalized Hecke eigenform of weight $2 k-n$ with respect to $S L_{2}(\mathbf{Z})$ corresponding to $h$ under the Shimura correspondence. For a prime number $p$ let $\beta_{p}$ be a non-zero complex number such that $\beta_{p}+\beta_{p}^{-1}=p^{-k+n / 2+1 / 2} c_{S(h)}(p)$. For a prime number $p$, let $\mathbf{Q}_{p}$, and $\mathbf{Z}_{p}$ be the field of $p$-adic numbers, and the ring of $p$-adic integers, respectively. We denote by $\nu_{p}$ the additive valuation on $\mathbf{Q}_{p}$ normalized so that $\nu_{p}(p)=1$, and by $\mathbf{e}_{p}$ the continuous homomorphism from the additive group $\mathbf{Q}_{p}$ to $\mathbf{C}^{\times}$such that $\mathbf{e}_{p}(x)=\mathbf{e}(x)$ for $x \in \mathbf{Z}\left[p^{-1}\right]$. For a positive definite half integral matrix $T$ of degree $n$ write $(-1)^{n / 2} \operatorname{det}(2 T)$ as $(-1)^{n / 2} \operatorname{det}(2 T)=\delta_{T} f_{T}^{2}$ with $\delta_{T}$ a fundamental discriminant and $\mathfrak{f}_{T}$ a positive integer. We then define the local Siegel series $b_{p}(T, s)$ by

$$
b_{p}(T, s)=\sum_{R \in S_{n}\left(\mathbf{Q}_{p}\right) / S_{n}\left(\mathbf{Z}_{p}\right)} \mathbf{e}_{p}(\operatorname{tr}(T R)) p^{-\nu_{p}\left(\mu_{p}(R)\right) s}(s \in \mathbf{C})
$$

for each prime number $p$, where $\mu_{p}(R)=\left[R \mathbf{Z}_{p}^{n}+\mathbf{Z}_{p}^{n}: \mathbf{Z}_{p}^{n}\right]$. Then there exists a polynomial $F_{p}(T, X)$ in $X$ such that

$$
b_{p}(T, s)=F_{p}\left(T, p^{-s}\right)\left(1-p^{-s}\right)\left(1-\left(\frac{\triangleright_{T}}{p}\right) p^{n / 2-s}\right)^{-1} \prod_{i=1}^{n / 2}\left(1-p^{2 i-2 s}\right)
$$

(cf. [Ki].) We then put

$$
c_{I_{n}(h)}(T)=c_{h}\left(\left|\mathfrak{\triangleright}_{T}\right|\right) \prod_{p}\left(p^{k-n / 2-1 / 2} \beta_{p}\right)^{\nu_{p}\left(\mathfrak{f}_{T}\right)} F_{p}\left(T, p^{-(n+1) / 2} \beta_{p}^{-1}\right) .
$$

We note that $c_{I_{n}(h)}(T)$ does not depend on the choice of $\beta_{p}$. Define a Fourier series $I_{n}(h)(Z)$ by

$$
I_{n}(h)(Z)=\sum_{T \in \mathcal{L}_{n}>0} c_{I_{n}(h)}(T) \mathbf{e}(\operatorname{tr}(T Z)) .
$$

In [I] Ikeda showed that $I_{n}(h)(Z)$ is a cuspidal Hecke eigenform in $S_{k}\left(S p_{n}(\mathbf{Z})\right)$ and its standard $L$-function $L\left(s, I_{n}(h), \mathrm{St}\right)$ is given by

$$
L\left(s, I_{n}(h), \mathrm{St}\right)=\zeta(s) \prod_{i=1}^{n} L(s+k-i, S(h)) .
$$


We call $I_{n}(h)$ the Duke-Imamoglu-Ikeda lift (D-I-I lift) of $h$. Now using the same argument as in the proof of Theorem 1 of $[\mathrm{I}-\mathrm{K}]$ we obtain the following. For the details see [Ka].

Theorem 4.1 Let $\chi$ be a primitive Dirichlet character mod N. Then we have

$$
\begin{aligned}
L^{*}(s, F, \chi)= & 2^{n s}\left\{c_{n} R\left(s, h, E_{n / 2+1 / 2}, \chi\right) \prod_{j=1}^{n / 2-1} L\left(2 s-2 j, S(h), \chi^{2}\right)\right. \\
& \left.+d_{n} c_{h}(1) \prod_{j=1}^{n / 2} L\left(2 s-2 j+1, S(h), \chi^{2}\right)\right\},
\end{aligned}
$$

where $c_{n}$ and $d_{n}$ are non-zero rational numbers depending only on $n$.

Now by the above theorem combined with Theorem 3.5 we obtain:

Theorem 4.2 Let $N$ be a square free odd integer, and $N=p_{1} \cdots p_{r}$ be the prime decomposition of $N$. For each $i=1, \cdots, r$ let $l_{i}=$ G.C.D $\left(n, p_{i}-1\right)$ and $u_{0} \in \mathbf{Z}$ be a primitive $l_{i}$-th root of unity $\bmod p_{i}$.

(1) Assume $\chi^{\left(p_{i}\right)}\left(u_{i}\right) \neq 1$ for some $i$. Then $L\left(s, I_{n}(h), \chi\right)=0$.

(2) Assume $\chi^{\left(p_{i}\right)}\left(u_{i}\right)=1$ for any $i$. Then

$$
\begin{gathered}
L\left(s, I_{n}(h), \chi\right)=2^{n s} \sum_{i_{1}=0}^{l_{1}-1} \cdots \sum_{i_{r}=0}^{l_{r}-1} \overline{\widetilde{\chi}_{\left(i_{1}, \cdots, i_{r}\right)}\left(2^{n}\right)} \overline{J\left(\widetilde{\chi}_{\left(i_{1}, \cdots, i_{r}\right)},\left(\frac{*}{N}\right)\right)} \overline{J_{n-1}\left(\widetilde{\chi}_{\left(i_{1}, \cdots, i_{r}\right)}\right)} \\
\quad \times\left\{c_{n, N} R\left(s, h, E_{n / 2+1 / 2}, \widetilde{\chi}_{\left(i_{1}, \cdots, i_{r}\right)}\right) \prod_{j=1}^{n / 2-1} L\left(2 s-2 j, S(h), \widetilde{\chi}_{\left(i_{1}, \cdots, i_{r}\right)}^{2}\right)\right. \\
\left.+d_{n, N} c_{h}(1) \prod_{j=1}^{n / 2} L\left(2 s-2 j+1, S(h), \widetilde{\chi}_{\left(i_{1}, \cdots, i_{r}\right)}^{2}\right)\right\},
\end{gathered}
$$

where $c_{n, N}$ and $d_{n, N}$ are non-zero rational numbers depending only on $n$ and $N$, and $\tilde{\chi}$ is a character s.t. $\tilde{\chi}^{n}=\chi$.

Remark. In the case $n=2$, an explicit formula for $L\left(s, I_{2}(h), \chi\right)$ was given by Katsurada-Mizuno [K-M]. 
Corollary Let $\chi$ be a Dirichlet character of odd square free conductor $N$ such that $\chi^{n}$ is primitive. Then for any integer $n / 2+1 \leq m \leq k-n / 2-1$

$$
\begin{gathered}
\frac{L\left(m, I_{n}(h), \chi^{n}\right)}{\pi^{m n}} \\
=\left\{\gamma_{n, N} \frac{R^{(\chi)}\left(m, h, E_{n / 2+1 / 2}\right)}{\pi^{m n}}+\delta_{n, N} c_{h}(1) \frac{\mathbf{M}^{(\chi)}(m, S(h))}{\pi^{m n}}\right\},
\end{gathered}
$$

where $\gamma_{n, N}$ and $\delta_{n, N}$ are non-zero numbers, and

$$
\begin{aligned}
\mathbf{M}^{(\chi)}(m, S(h))= & \left.\sum_{i_{1}=0}^{l_{1}-1} \cdots \sum_{i_{r}=0}^{l_{r}-1} \overline{\chi_{\left(i_{1}, \cdots, i_{r}\right)}\left(2^{n}\right)} \overline{J\left(\chi_{\left(i_{1}, \cdots, i_{r}\right)},\left(\frac{*}{N}\right)\right)} \overline{J_{n-1}\left(\chi_{\left(i_{1}, \cdots, i_{r}\right)}\right.}\right) \\
& \times \prod_{j=1}^{n / 2} L\left(2 m-2 j+1, S(h),\left(\chi_{\left(i_{1}, \cdots, i_{r}\right)}\right)^{2}\right) .
\end{aligned}
$$

\section{Proof of main results and some comments}

We prove the results in Section 2.

Proof of Theorem 2.1. Assume that $n \equiv 2 \bmod 4$. Then we have $c_{h}(1)=0$, and by Theorem 3.1 and Corollary to Theorem 4.2, we have

$$
\frac{R^{(\chi)}\left(m, h, E_{n / 2+1 / 2}\right)}{\pi^{m n}} \in \overline{\mathbf{Q}} u_{1} \otimes_{\overline{\mathbf{Q}}} V_{I_{n}(h)}
$$

with some complex number $u_{1}$, where $V_{I_{n}(h)}$ is the $\overline{\mathbf{Q}}$-vector space associated with $I_{n}(h)$ in Theorem 3.1. Assume that $n \equiv 0 \bmod 4$. By Theorem 1.1 we have

$$
\frac{\mathbf{M}^{(\chi)}(m, S(h))}{\pi^{m n}} \in \overline{\mathbf{Q}} u_{-}(S(h))^{n / 2} \pi^{-n^{2} / 4} .
$$

Hence, again by Theorem 3.1 and Corollary to Theorem 4.2,

$$
\frac{R^{(\chi)}\left(m, h, E_{n / 2+1 / 2}\right)}{\pi^{m n}} \in \overline{\mathbf{Q}} u_{1} \otimes_{\overline{\mathbf{Q}}} V_{I_{n}(h)}+\overline{\mathbf{Q}} u_{2}
$$

with complex numbers $u_{1}$ and $u_{2}$. This proves the assertion. 
Proof of Theorem $\mathbf{2 . 2}$ and its corollary. Theorem 2.2 follows directly from Theorem 2.1. We note that $J_{n-1}\left(\chi_{\left(i_{1}, \cdots, i_{r}\right)}\right)$ is a non-zero algebraic number by virtue of Corollary to Proposition 3.8. We also note that $\frac{\mathbf{L}_{n}(m, S(h), \eta)}{\pi^{m(n-2)}}$ belongs to $\overline{\mathbf{Q}} u_{+}(S(h))^{n / 2-1} \pi^{-n^{2} / 4+n / 2}$, and nonzero for any integer $n / 2+1 \leq$ $m \leq k-n / 2-1$ and primitive character $\eta$. This proves the corollary.

Proof of Theorem 2.3. The assertion follows from Theorem 3.2.

Now we give some comments. First we are interested in the dimension of $W_{h, E_{n / 2+1 / 2}}$ over $\overline{\mathbf{Q}}$. Therefore we propose the following problem.

Problem 1. Give $\operatorname{dim}_{\overline{\mathbf{Q}}} W_{h, E_{n / 2+1 / 2}}$ explicitly or estimate it.

This problem is reduced to the following problem:

Problem 2. Give $\operatorname{dim}_{\overline{\mathbf{Q}}} V_{I_{n}(h)}$ explicitly or estimate it.

Next we consider a generalization or a refinement of Theorem 2.1. Namely we propose the following conjecture.

Conjecture. Let $h_{1}(z)$ be a Hecke eigenform in $S_{k_{1}+1 / 2}^{+}\left(\Gamma_{0}(4)\right)$ and $h_{2}(z) \in$ $M_{k_{2}+1 / 2}\left(\Gamma_{0}(4)\right)$ with $k_{1} \geq k_{2}+2$. Assume that $c_{h_{2}}(m) \in \overline{\mathbf{Q}}$ for any $m \in \mathbf{Z}_{\geq 0}$. Then there exists a finite dimensional $\overline{\mathbf{Q}}$-vector space $W_{h_{1}, h_{2}} \subset \mathbf{C}$ such that

$$
R\left(m, h_{1}, h_{2}, \chi\right) \pi^{-2 m} \in W_{h_{1}, h_{2}}
$$

for any $k_{2}+1 \leq m \leq k_{1}-1$ and any primitive character $\chi$.

Problem 3. Prove Theorem 2.1 without using the relation between the twisted Koecher-Maaß series of the Duke-Imamoglu-Ikeda lift and the twisted Rankin-Selberg series of modular forms of half-integral weight.

\section{References}

[C-K] Y. Choie and W. Kohnen, Special values of Koecher-Maaß series of Siegel cusp forms, Pacific J. Math. 198 (2001), 373-383.

[I] T. Ikeda, On the lifting of elliptic modular forms to Siegel cusp forms of degree 2n, Ann. of Math. 154(2001), 641-681. 
[I-K] T. Ibukiyama and H. Katsurada, An explicit formula for KoecherMaaß Dirichlet series for the Ikeda lifting, Abh. Math. Sem. Hamburg 74(2004), 101-121.

[Ka] H. Katsurada, Explicit formulas of twisted Koecher-Maaß series of the Duke-Imamoglu-Ikeda lift and their applications, To appear in Math. Z.

[K-M] H. Katsurada and Y. Mizuno, Linear dependence of certain L-values of half-integral weight modular forms, J. London Math. 85(2012), 455471.

[Ki] Y. Kitaoka, Dirichlet series in the theory of Siegel modular forms, Nagoya Math. J. 95(1984), 73-84.

[Ko] W. Kohnen, New forms of half-integral weight, J. reine und angew. Math. 333(1982) 32-72.

[Sh1] G. Shimura, On the periods of modular forms, Math. Ann. 229(1977), 211-221.

[Sh2] G. Shimura, The critical values of certain zeta functions associated with modular forms of half-integral weight, J. Math. Soc. Japan 33(1981), 649-672.

Hidenori KATSURADA

Muroran Institute of Technology

27-1 Mizumoto, Muroran, 050-8585, Japan

E-mail: hidenori@mmm.muroran-it.ac.jp 